\title{
Universiteit
}

Leiden

The Netherlands

\section{Elastic lattice polymers}

Baiesi, M.; Barkema, G.T.; Carlon, E.

\section{Citation}

Baiesi, M., Barkema, G. T., \& Carlon, E. (2010). Elastic lattice polymers. Physical Review E, 81(6), 061801. doi:10.1103/PhysRevE.81.061801

Version: $\quad$ Not Applicable (or Unknown)

License: $\quad$ Leiden University Non-exclusive license

Downloaded from: https://hdl.handle.net/1887/61287

Note: To cite this publication please use the final published version (if applicable). 


\title{
Elastic lattice polymers
}

\author{
M. Baiesi, ${ }^{1,2}$ G. T. Barkema, ${ }^{2,3,4}$ and E. Carlon ${ }^{2}$ \\ ${ }^{1}$ Department of Physics, University of Padova, via Marzolo 8, 35131 Padova, Italy \\ ${ }^{2}$ Institute for Theoretical Physics, K. U. Leuven, Celestijnenlaan 200D, B-3001 Leuven, Belgium \\ ${ }^{3}$ Institute for Theoretical Physics, Utrecht University, Leuvenlaan 4, 3584CE Utrecht, The Netherlands \\ ${ }^{4}$ Instituut-Lorentz, Universiteit Leiden, Niels Bohrweg 2, 2333 CA Leiden, The Netherlands
}

(Received 22 February 2010; published 4 June 2010)

\begin{abstract}
We study a model of "elastic" lattice polymer in which a fixed number of monomers $m$ is hosted by a self-avoiding walk with fluctuating length $l$. We show that the stored length density $\rho_{m} \equiv 1-\langle l\rangle / m$ scales asymptotically for large $m$ as $\rho_{m}=\rho_{\infty}(1-\theta / m+\ldots)$, where $\theta$ is the polymer entropic exponent, so that $\theta$ can be determined from the analysis of $\rho_{m}$. We perform simulations for elastic lattice polymer loops with various sizes and knots, in which we measure $\rho_{m}$. The resulting estimates support the hypothesis that the exponent $\theta$ is determined only by the number of prime knots and not by their type. However, if knots are present, we observe strong corrections to scaling, which help to understand how an entropic competition between knots is affected by the finite length of the chain.
\end{abstract}

DOI: 10.1103/PhysRevE.81.061801

PACS number(s): 36.20.Ey, 02.10.Kn, 87.15.A-

\section{INTRODUCTION}

According to renormalization group theory, the scaling properties of critical systems are insensitive to microscopic details and are governed by a small set of universal exponents [1]. Also polymers can be considered as critical systems in the limit where their length $l$ (the number of chained monomers) diverges [2-4]. For instance, the radius of gyration of an isolated polymer in a swollen phase scales as $R_{g}$ $\sim l^{\nu}$, where $\nu \approx 0.587597(7)$ [5] in $d=3$ dimensions is a universal critical exponent. One of the simplest models in the universality class of swollen polymers is that of selfavoiding walks (SAWs) on a lattice. Hence, these have been used extensively to extract information on critical exponents and scaling functions $[2,4-23]$. The total number of SAWs, i.e., their partition function, has the following large- $l$ expansion

$$
Z_{l} \sim \mu^{l} l^{\theta}\left(1+A l^{-\Delta}+\ldots\right) .
$$

Here, nonuniversal (model-dependent) quantities are the connectivity constant $\mu$ and the amplitude of the corrections to scaling A. The entropic exponent $\theta$ depends only on boundary conditions: in $d=3$ we have $\theta \equiv \gamma-1=0.1573$ (2) [24] for an open chain whereas $\theta \equiv \alpha-2=-d \nu=-1.762791(21)$ [5] for self-avoiding polygons (SAPs), that is, linear chains with the two ends on adjacent lattice sites. Renormalization group analysis suggests that the exponent $\Delta$, characterizing the leading corrections to the scaling behavior, is also universal $[1,3]$

Models with full self-avoidance, such as SAPs, have been used to study the statistical properties of knotted chains $[13,14,19,25-35]$. Knots in polymers have attracted a lot of attention during the past years, also because of their occurrence in biopolymers as DNAs, RNAs, and proteins [36-41]. As usual, SAWs represent a minimal effective model to grasp the essential, coarse-grained features of polymer chains. Simulations of knotted SAPs in ensembles with fixed topology are performed with a grand-canonical algorithm (BFACF [6], from the name of the authors) tuned to span a range of chain lengths (algorithms with fixed $N$ are not ergodic in this case). For this algorithm, the tuning of step fugacities to $u \approx 1 / \mu$ is necessary to achieve samplings of long chains. It would be desirable to have a simpler and more stable method to sample the same chain lengths.

In this paper, we study a class of polymers referred to as the elastic lattice polymers (ELPs), which are SAWs accumulating some stored length along their contour. This leads in fact to a partial lifting of the self-avoidance condition between consecutive monomers of an ELP, as sketched in Fig. 1. We will consider equilibrium properties of polymers with a fixed number of monomers $m$, and in which as a consequence the length $l \leq m$ of the self-avoiding backbone described by the monomers fluctuates. This explains the name "elastic," and implies a resemblance with the class of grand-canonical SAW models.

There are several reasons for studying this model. On the theoretical side, it can be considered as an enhanced SAW: besides sharing critical exponents with SAWs, its fluctuating length enables new avenues to estimate critical exponents. ELPs have been used in studies of polymer dynamics as phase separation in polymer melts [22], or in translocation through nanopores [21], but their equilibrium properties have so far received little attention. The key quantity we focus on

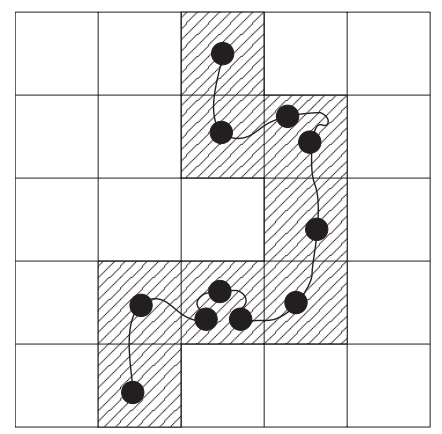

FIG. 1. Example of an elastic lattice polymer on a square lattice. This polymer is composed by $m=11$ monomers describing a SAW backbone of length $l=8$ (dashed area). 
is the equilibrium averaged stored length density defined as

$$
\rho_{m} \equiv \frac{m-\langle l\rangle}{m},
$$

where $\langle l\rangle$ depends on $m$. As will be shown, $\rho_{m}$ has a simple asymptotic behavior for large $m$ from which one can extract universal exponents: the leading correction to the asymptotic value for $\rho_{m}$ scales as $\theta / m$, where $\theta$ is the entropic exponent defined by Eq. (1). We illustrate the result of this approach for the case of ELPs with fixed knots. If knots are present, the stored length approaches its asymptotic value with strong, knot-dependent corrections to scaling. The expectation of a homogeneous stored length within an equilibrated chain, combined with the knowledge on how its density varies with the chain length, leads us to a new view on the issue of entropic competition of knotted regions [25]. On the numerical side, we find that ELPs, compared to grandcanonical algorithms, have the nice feature of stabilizing the sampling quite narrowly around an easily tunable length $\langle l\rangle$.

This paper is organized as follows. In Section II, we derive the expansion for $\rho_{m}$ as a function of $m$. In Section III, we illustrate how to estimate entropic exponents via fits of $\rho_{m}$, with a reweighting of exact enumeration data for polymers on square and cubic lattices. In Section IV, we present Monte Carlo simulations of ELPs containing a fixed knot and determine the averaged stored length in equilibrium as a function of $m$. The entropic exponent $\theta$ is determined for different simple and double knots. Finally, in Section V, we discuss, on the basis of the obtained scaling behavior for $\rho_{m}$, different possible scenarios for knot competitions.

\section{SCALING PROPERTIES OF THE STORED LENGTH}

Consider a polymer composed by $m$ monomers with lattice coordinates defined by $\vec{r}_{i}, i=1,2, \ldots m$. Multiple occupancy of neighboring monomers on the same lattice site means that we allow configurations for which $\vec{r}_{k}=\vec{r}_{k+1}=\ldots$ $=\vec{r}_{k+p}=\vec{s}$. However if $\vec{r}_{k-1} \neq \vec{s}$ and $\vec{r}_{k+p+1} \neq \vec{s}$, then no monomers other than those of the interval $[k, k+p]$ are allowed to visit the site $\vec{s}$. The lattice polymer so defined describes a self-avoiding backbone of length $0 \leq l \leq m$. The two extremal cases are all monomers occupying the same lattice point $(l=0)$ and a fully stretched configuration without multiple occupancy $(l=m)$. The equilibrium partition function for an ELP with $m$ monomers is given by

$$
\tilde{Z}_{m}=\sum_{l=0}^{m}\left(\begin{array}{c}
m \\
l
\end{array}\right) u^{l} Z_{l},
$$

where the sum is over the length $l$ of the self-avoiding backbone and $Z_{l}$ is the canonical partition function, which counts the number of allowed configurations for the self-avoiding backbone, and whose asymptotic is given in Eq. (1). The factor $\left(\begin{array}{c}m \\ l\end{array}\right)$ in Eq. (3) counts the number of ways the stored length can be distributed over the backbone. For convenience an extra fugacity $u$ per site has been added.

Substituting Eq. (1) in Eq. (3) and defining $\omega=\mu u$, the average backbone length $\langle l\rangle$ can be computed from

$$
\langle l\rangle=\omega \frac{\partial}{\partial \omega} \log \widetilde{Z}_{m} .
$$

It is instructive to consider first the case of a partition function of the type $Z_{l}=\mu^{l}$ in Eq. (3), i.e., neglecting powerlaw and correction to scaling terms in Eq. (1). In this case Eq. (3) becomes

$$
\tilde{Z}_{m}^{\infty}=\sum_{l=0}^{m}\left(\begin{array}{c}
m \\
l
\end{array}\right) \omega^{l}=(1+\omega)^{m} .
$$

Equation (5) has the following interpretation: the partition function for a walk of $m$ steps factorizes as each monomer can either sit on the backbone (accumulating stored length with weight 1 ) or occupy a free site (with average weight $\omega$ ). From Eq. (4) we get the following value of the averaged backbone length

$$
l_{\infty}=\frac{m \omega}{1+\omega} .
$$

We now go back to the full partition function in Eq. (3). For large $m$ and fixed $\omega$ the binomial factor is sharply peaked around $l=l_{\infty}$. We approximate the binomial by a Gaussian distribution as follows:

$$
\left(\begin{array}{c}
m \\
l
\end{array}\right) \omega^{l} \approx(1+\omega)^{m} \frac{1}{\sqrt{2 \pi \sigma^{2}}} e^{-\left(l-l_{\infty}\right)^{2} / 2 \sigma^{2}},
$$

where

$$
\sigma^{2}=\frac{m \omega}{(1+\omega)^{2}} .
$$

The Gaussian approximation differs from the binomial by terms which are exponentially small for large $m$, which are of higher order in the large- $m$ expansion we are interested in, so they can be safely neglected. We replace now the discrete sum in Eq. (3) by an integral over all lengths, extending the domain of integration in the whole real axis:

$$
\tilde{Z}_{m}=\frac{(1+\omega)^{m}}{\sqrt{2 \pi \sigma^{2}}} \int_{-\infty}^{+\infty} d l e^{-\left(l-l_{\infty}\right)^{2} / 2 \sigma^{2}} l^{\theta}\left(1+A l^{-\Delta}\right),
$$

where we have replaced the asymptotic form of $Z_{l}$ as given in Eq. (1). The replacement of the sum by an integral brings corrections in Eq. (9), which are of higher order in $1 / m$ and for our purposes can be neglected.

We solve the integral in Eq. (9) by using a saddle point approximation. A simple rescaling $l=x l_{\infty}$ gives

$$
\tilde{Z}_{m}=(1+\omega)^{m} \sqrt{\frac{m \omega}{2 \pi}} \int_{-\infty}^{+\infty} d x e^{m \omega \Gamma(x)}
$$

with

$$
\Gamma(x)=\frac{-(x-1)^{2}}{2}+\frac{\theta \log \left(x l_{\infty}\right)+\log \left(1+A\left(x l_{\infty}\right)^{-\Delta}\right)}{m \omega} .
$$

Let $\bar{x}$ the maximum of $\Gamma(x)$. We have: 


$$
\begin{aligned}
\tilde{Z}_{m} & \approx(1+\omega)^{m} \sqrt{\frac{m \omega}{2 \pi}} e^{m \omega \Gamma(\bar{x})} \sqrt{\frac{2 \pi}{m \omega\left|\Gamma^{\prime \prime}(\bar{x})\right|}} \\
& =(1+\omega)^{m} \frac{e^{m \omega \Gamma(\bar{x})}}{\sqrt{\left|\Gamma^{\prime \prime}(\bar{x})\right|}} .
\end{aligned}
$$

Equation (11) implies that the maximum of $\Gamma(x)$ in the large- $m$ limit is $\bar{x}=1+\mathcal{O}(1 / m)$, giving

$$
\left|\Gamma^{\prime \prime}(\bar{x})\right|=1+\mathcal{O}\left(\frac{1}{m}\right),
$$

which produces higher-order terms, which we neglect in the large- $m$ expansion. In addition:

$$
m \omega \Gamma(\bar{x})=\theta \log l_{\infty}+\log \left(1+A l_{\infty}^{-\Delta}\right)+\ldots
$$

Equations (12)-(14) again show that the leading contribution to the partition function $\widetilde{Z}_{m}$ is $(1+\omega)^{m}$, but also that the subleading contribution $\sim l_{\infty}^{\theta} \sim m^{\theta}$ has the same entropic exponent $\theta$ of SAWs. From Eq. (4) we get

$$
\langle l\rangle=l_{\infty}\left[1+\frac{\theta}{m \omega}-\frac{A}{m \omega}\left(\frac{1+\omega}{m \omega}\right)^{\Delta}\right]
$$

and the stored length density Eq. (2) becomes

$$
\rho_{m}=\rho_{\infty}\left[1-\frac{\theta}{m}+\left(\frac{1+\omega}{\omega}\right)^{\Delta} \frac{A}{m^{1+\Delta}}\right],
$$

where we defined

$$
\rho_{\infty}=\frac{1}{1+\omega} .
$$

The expansion Eq. (16) is valid provided $\Delta<1$. The neglected terms coming from the replacement of the sum with an integral, and from the Gaussian integration in Eq. (10), are of the order $1 / \mathrm{m}^{2}$ (except if $\Delta>1$, the $\mathcal{O}\left(1 / \mathrm{m}^{2}\right.$ ) terms would dominate over the $1 / m^{1+\Delta}$.) The value of the exponent $\theta$ can then be obtained from a plot of $\rho_{m}$ vs $1 / m$, as the slope in the limit $1 / m \rightarrow 0$. Using the high-precision literature values for the connectivity constants $\mu$, one obtains a very accurate estimate of $\rho_{\infty}$.

With the definition of $\rho_{\infty}$ in Eq. (17) we can rewrite the variance Eq. (8) as

$$
\sigma^{2}=m \rho_{\infty}\left(1-\rho_{\infty}\right)
$$

This form reveals clearly that the largest $\sigma$ for a given $m$ is achieved with $\rho_{\infty}=1 / 2$, i.e., with a fugacity $u=\mu^{-1}$. We can think of this regime as the maximally elastic one. In all cases, note that the relative polydispersity $\sigma / m$ of the chains goes to zero $\sim m^{-1 / 2}$ for $m \rightarrow \infty$, hence the chain lengths $l$ are narrowly distributed around their average $\langle l\rangle$. This allows us to use saddle-point approximations (see Sec. V) and leads to metric properties in the universality class of SAWs (e.g., radius of gyration scaling as $\sim m^{\nu} \sim\langle l\rangle^{\nu}$ ). Hence, ELPs share both exponents $\theta$ and $\nu$ with SAWs.
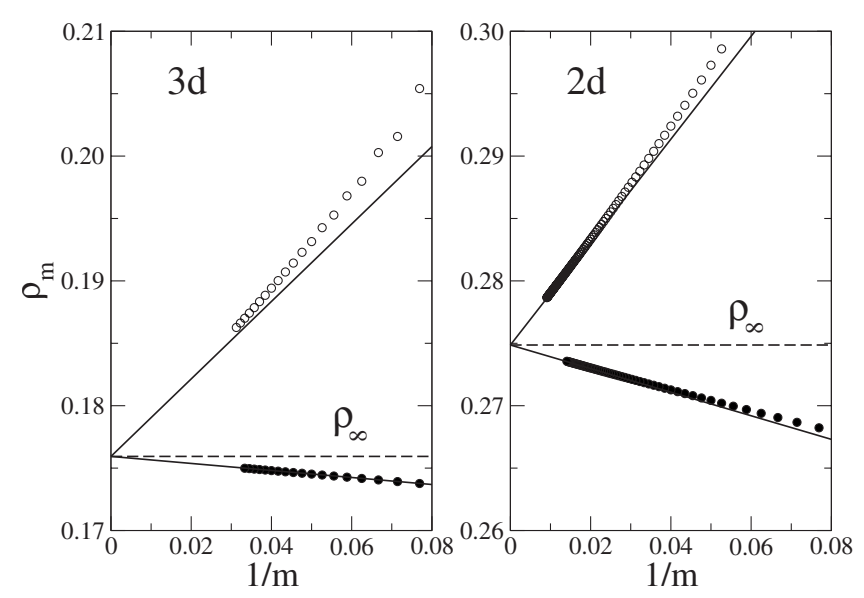

FIG. 2. Stored length calculated from exact enumeration data for SAWs (bullets) and SAPs (empty circles) on the cubic lattice $(d=3)$ and on the square lattice $(d=2)$. The solid lines are the leading terms in the $\rho_{m}$ vs $1 / m$ expansion when using the expected value for the exponent $\theta$. The asymptotic values $\rho_{\infty}=1 /(1+\mu)$ for cubic and square lattices are shown as a dashed line.

\section{STORED LENGTH FROM EXACT ENUMERATIONS DATA}

As a first illustration of the scaling behavior of the stored length $\rho_{m}$ as a function of the number of monomers $m$, we consider exact enumeration data for SAWs and SAPs on square and cubic lattices, which are taken from the published literature [17]. Enumeration techniques provide exact values for the total number of SAWs $Z_{l}$ as a function of their length $l$. We use these values for $Z_{l}$ to compute $\widetilde{Z}_{m}$ from Eq. (3). The stored length $\rho_{m}$ is obtained from the average $\langle l\rangle$, using Eq. (2). We have the freedom to choose the value of the fugacity $u$ in Eq. (3).

Figure 2 shows a plot of $\rho_{m}$ as a function of $1 / m$ for three and two dimensions, obtained by setting $u=1$ in Eq. (3). The data converge to the expected asymptotic value, which is $\rho_{\infty} \simeq 0.175931$ (cubic) and $\rho_{\infty} \simeq 0.2748643$ (square). These are obtained from Eq. (17) with $\omega=u \mu=\mu$ and the following values for the connectivity constants: $\mu=4.684044(11)$ [20] (cubic) and $\mu=2.63815852927(1)$ (square) [16].

The solid lines in Fig. 2 are the linear terms in the expansion of Eq. (16) where the value of $\theta$ is that for open walks $(\theta=11 / 32$ in $d=2$ and $\theta \simeq 0.157$ in $d=3)$ and polygons $(\theta=-3 / 2$ in $d=2$ and $\theta \simeq-1.76$ in $d=3)$. The results show that the linear scaling in $1 / \mathrm{m}$ sets in already for short polymers $(m \approx 20)$. In addition we observe that the corrections to the leading scaling behavior are stronger for closed walks (empty circles) compared to the open walks case (filled circles).

We performed finite-size extrapolations to obtain estimates of $\theta$ from $\rho_{m}$. The two-dimensional data have been extrapolated by means of the Burlisch-Stoer (BST) algorithm [42], using the finite- $m$ approximants

$$
\theta_{m} \equiv \frac{\rho_{m}-\rho_{m-1}}{\rho_{m-1} / m-\rho_{m} /(m-1)},
$$

which are the ratios between slope and intercept of the line joining the points $\left(1 /(m-1), \rho_{m-1}\right)$ and $\left(1 / m, \rho_{m}\right)$, i.e., they 
TABLE I. Summary of the exponents obtained from the extrapolation of the approximants $\theta_{m}$ defined in Eq. (19). The data are for SAWs and SAPs [17]. The last column gives the exact two dimensional data [4].

\begin{tabular}{lccc}
\hline \hline Type & Max $m[17]$ & $\theta$ & $\theta_{\mathrm{ex}}$ \\
\hline SAW, $d=2$ & 71 & $0.3437(2)$ & $0.34375\left(=\frac{11}{32}\right)$ \\
SAP, $d=2$ & 110 & $-1.500(1)$ & $-\frac{3}{2}$ \\
SAW,$d=3$ & 30 & $0.158(2)$ & \\
SAP, $d=3$ & 32 & $-1.75(2)$ & \\
\hline
\end{tabular}

are finite-size estimates of the ratio between $\rho_{\infty} \theta$ and $\rho_{\infty}$. The BST algorithm starts with a sequence of $N$ elements, and generates iteratively sequences of $N-1, N-2 \ldots$ elements which are expected to converge faster at each iteration step. It involves a free parameter $(\Omega)$, which roughly measures the effective leading correction exponent. In our extrapolations, an optimal value of $\Omega$ was selected requiring a minimal standard deviation of the last five sequences generated by the iterative algorithm. The extrapolations were repeated for different values of the fugacity parameter $u$ and the error was estimated from the variation on these values. For threedimensional data, the BST algorithm turned out not to be very accurate, particularly for loops. The reason is that $\rho_{m}$ for small $m$ has some subleading oscillatoric behavior which is not sufficiently damped during the BST iterations. The result is that the accuracy of the extrapolation is poor. For these data we use instead a nonlinear fit, fixing $\rho_{\infty}$ and keeping $\theta$, $A$, and $\Delta$ as fitting parameters.

The extrapolated values for $\theta$ are reported in Table I; these are accurate and in good agreement with exact data in two dimensions and also with the best numerical estimates in three dimensions $(\theta=0.1573(2)$ [24] for walks, $\theta$ $=-1.76279(2)$ for polygons-assuming hyperscaling $\alpha-2$ $=-d \nu$ with $\nu=0.587597(7)$ [5]), which shows that reliable values of the entropic exponents can be extracted from the scaling of the stored length.

\section{ENTROPIC EXPONENTS OF KNOTTED POLYMERS}

We now turn to the study of equilibrium properties of ELP rings with some fixed topology. Here, we will show how the knowledge of the stored length $\rho_{m}$ can be exploited to investigate equilibrium properties of knotted polymers.

We have performed Monte Carlo simulations of ELPs on the face-centered-cubic (fcc) lattice, with an algorithm that was recently used to study translocation dynamics [21] and phase separation in polymer melts [22]. The allowed Monte Carlo update moves include reptation, i.e., the diffusion of stored length along its backbone and Rouse-like moves, which locally change the backbone configuration. (For more details see Ref. [22].).

The setup of the simulation is as follows. We start from a backbone with a minimal number of steps on the fcc lattice, as those shown in Fig. 3. A total number of monomers $m$ are distributed randomly over this backbone. These configurations are then relaxed to equilibrium. Typically $m$ is much

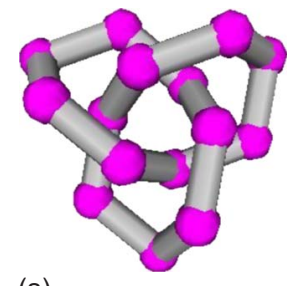

(a)

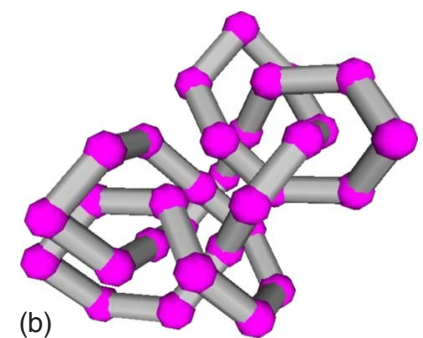

FIG. 3. (Color online) Examples of knots on the fcc lattice: (a) a 3 knot (trefoil) with $l=15$ steps and (b) a composite $3_{1} \# 4_{1}$ knot with $l=31$ steps (the notation $k_{1} \# k_{2}$ indicates a closed polymer ring with two knots, one of type $k_{1}$ and one of type $k_{2}$ ). These configurations have been used as backbones with stored length $m-l$ for starting the simulations of ELP with $m \geq l$ monomers.

larger than the initial length (we simulated polymers with $m$ up to 2000) so that relaxation to equilibrium corresponds to an expansion of the backbone. The Monte Carlo moves preserve the knot topology imposed initially. Once equilibrium is reached we start the sampling of the stored length density $\rho_{m}$.

An additional weight (equal to 4) is introduced for moves that accumulate monomers on the same lattice point, which corresponds to a fugacity factor $u=1 / 4$ in Eq. (3). This leads to the following asymptotic value for the stored length density:

$$
\rho_{\infty}=\frac{1}{1+\mu / 4}=0.28498(1)
$$

where the numerical value is obtained by considering the most accurate available estimate $\mu=10.0362(6)$ [9] for the connectivity constant of SAWs on fcc lattice [47].

Figure 4 shows the scaling behavior of $\rho_{m}$ as a function of $1 / m$ for an unknotted polymer ring, for single and double knots. All data converge asymptotically to the value $\rho_{\infty}$ obtained from Eq. (20). This value is shown as a dashed horizontal line in Fig. 4 . We note that the approach to $\rho_{\infty}$ of the numerical data for unknotted rings is quite different for those of rings with knots (a detail of the asymptotic region is shown in the inset of Fig. 4): the data for the unknotted topology approach the asymptotic value with a clear $1 / \mathrm{m}$ scaling behavior. For topologies with knots instead there is a pronounced curvature in the $\rho_{m}$ vs $1 / m$ plot, deriving from strong corrections to scaling. These corrections are stronger for an increasing knot complexity and for an increasing number of knots. The shortest length $l_{\min }$ of a knot on a lattice is a good indicator of its complexity, and in this model for $m$ $=l=l_{\min }$ by definition the chain can only be fully stretched, i.e., $\rho_{m_{\min }}=0$. Our data show that the crossover from this initial topological stretching to the asymptotic regime $\sim m^{-1}$ grows quickly with the value of $m_{\text {min }}$. In this view, the fact that for unknotted chains on the fcc lattice one has $m_{\min }=3$, much smaller than that of the simplest knot (the trefoil with $m_{\min }=15$ ) explains why corrections to scaling are negligible for unknotted chains.

We estimated the entropic exponent $\theta$ using the scaling behavior predicted by Eq. (16). In the unknotted case due to 


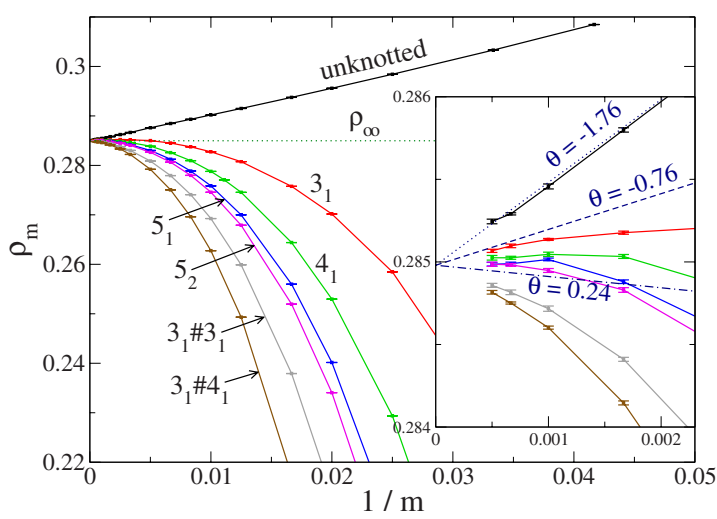

FIG. 4. (Color online) Plot of the average equilibrium stored length density $\rho_{m}$ as a function of the inverse monomer number $1 / m$ for closed polymers with some fixed topology. The simulations were extended to polymers of lengths up to $m=2000$. From top to bottom the data refers to: unknotted ring, $3_{1}, 4_{1}, 5_{1}$, and $5_{2}$ knots. The two bottom data set correspond to configuration with two knots: $3_{1} \# 3_{1}$ and $3_{1} \# 4_{1}$, respectively. The horizontal dashed line is $\rho_{\infty}=0.28498$, as expected from Eq. (20). Inset: zoom of the asymptotic region. Straight lines represent $\rho_{\infty}(1-\theta / m)$ : the dotted line corresponds to the conjectured value $\theta=-3 \nu$, the dashed line to $\theta=-3 \nu+1$, and the dot-dashed line to $\theta=-3 \nu+2$.

the manifest absence of curvature of the data, we restricted ourselves to a linear fit setting $A=0$ and $\rho_{\infty}=0.28498$ in Eq. (16) and using $\theta$ as the only free parameter. The fit, restricted to $m \geq 200$, yields $\theta=-1.76(3)$, confirming that the entropic exponent for rings with fixed unknotted topology is identical to that for SAPs with no topological constraints.

A closer look at the data reveals that the stored length density for knots $3_{1}, 4_{1}$ and $5_{1}$ is nonmonotonic. As the data asymptotically approach $\rho_{\infty}$ from above, Eq. (16) implies a negative value of the exponent $\theta$. We performed a nonlinear three-parameters fit to the databased on Eq. (16): $\theta, \Delta$ and $A$ are fitting parameters while we fix $\rho_{\infty}=0.28498$, as predicted by Eq. (20). The results of the nonlinear fits are given in Table II. The estimated exponent changes sign from single knot $(\theta<0)$ to double knots $(\theta>0)$. A range of correctionto-scaling exponents $\Delta$ providing optimal fits were selected and these are given in the third column of Table II. Error

TABLE II. Summary of the estimated entropic exponents obtained from the scaling behavior of the stored length with a three parameters fit $\left[\theta, \Delta\right.$ and $A$ in Eq. (16)]. The asymptotic value $\rho_{\infty}$ is kept fixed. The last column shows the range of polymer sizes used in the fit.

\begin{tabular}{lccc}
\hline \hline Knot type & $\theta$ & $\Delta$ & Range of $m$ \\
\hline Unknotted & $-1.76(3)$ & & $\geq 200$ \\
$3_{1}$ & $-0.75(5)$ & $0.5-0.7$ & $\geq 400$ \\
$4_{1}$ & $-0.6(1)$ & $0.9-1.2$ & $\geq 300$ \\
$5_{1}$ & $-0.5(2)$ & $0.9-1.2$ & $\geq 300$ \\
$5_{2}$ & $-0.3(3)$ & $0.9-1.2$ & $\geq 300$ \\
$3_{1} \# 3_{1}$ & $0.4(2)$ & $1.2-1.4$ & $\geq 300$ \\
$3_{1} \# 4_{1}$ & $0.8(2)$ & $1.2-1.4$ & $\geq 300$ \\
\hline \hline
\end{tabular}

estimates for $\theta$ reflect the variability in $\theta$ from the different values of $\Delta$ used in the analysis. For the knots studied, the most accurate estimate for $\theta$ is that of the $3_{1}$ knot, yielding $\theta=-0.75(5)$. The error increases with the knot complexity. For single knots we also note a change in the range of correction-to-scaling exponents from $\Delta \approx 0.6$ for the 3 knot to $\Delta \approx 1.1$ in the other knots.

It has been suggested $[13,14,35]$ that for a knot $k$ with $\pi_{k}$ prime components, the entropic exponent is given by $\theta_{k}=\theta$ $+\pi_{k}$, where $\theta$ is the exponent for a polymer ring without fixed topology. If this is the case we expect for a single knot an exponent $\theta=-3 \nu+1=-0.76$ while for double knots $\theta=-3 \nu+2=0.24$ (these conjectured values are shown as dashed lines in the inset of Fig. 4). The idea behind this suggested scaling is that localized knots are like sliding entities, which can occupy any of the $l$ sites of a chain, thus contributing entropically with a factor $l$ in the partition function. Our numerical results fully support this conjecture for the single $3_{1}$ knot and also for the double $3_{1} \# 3_{1}$ knot. Results for the other knots seem to overestimate $\theta$ with respect to the conjectured values. It is likely that the deviations from the conjectured values are due to strong finite-size effects. An indication of this is the value of the correction-to-scaling exponent obtained from the fits, which, with the exception of the $3{ }_{1} \mathrm{knot}$, is estimated as $\Delta \approx 1$. Renormalization group arguments [1] for magnetic $O(N)$ models, which map into polymer models in the limit $N \rightarrow 0$ [2], predict instead $\Delta$ $\approx 0.55$, and Clisby [5] finds $\Delta \approx 0.528(12)$ in simulations of very long SAWs (this is in agreement with the range of values obtained in the extrapolations of the numerical data for the $3_{1}$ knot, see Table II). We also remark that a value $\Delta$ $>1$ is at odds with the expansion of the stored length of Eq. (16) in which it was implicitly assumed $\Delta<1$, the $1 / m^{1+\Delta}$ term would be otherwise dominated by $\mathcal{O}\left(1 / \mathrm{m}^{2}\right)$ corrections, which were neglected in the computation of $\rho_{m}$ leading to Eq. (16).

In the sequel we fix the entropic exponents to the conjectured values $\bar{\theta} \equiv-3 \nu+\pi_{k}$ and subtract from $\rho_{m}$ the constant and leading correction in $1 / \mathrm{m}$ as

$$
f_{m} \equiv \rho_{m}-\rho_{\infty}\left(1-\frac{\bar{\theta}}{m}\right) .
$$

For this quantity we expect the following scaling behavior

$$
f_{m} \simeq \frac{\bar{A}}{m^{1+\Delta}}+\frac{\bar{B}}{m^{2}},
$$

where a next-order $1 / \mathrm{m}^{2}$ term has been added.

Figure 5 plots $f_{m} m^{1+\Delta}$, where we set $\Delta=0.5$, as a function of $m$. The fact that this quantity approaches a constant value for large $m$ supports an estimate of the correction-to-scaling exponent $\Delta \approx 0.5$, as expected for swollen polymers [4]. In addition the constant $\bar{A}$ is negative and its magnitude quickly increases with knot complexity. This is also visible in Fig. 4 as the effect of increasing $A$ is that of producing an increased curvature in a plot of $\rho_{m}$ vs $1 / \mathrm{m}$. It is perhaps not surprising that finite-size effects increase with the knot complexity, as more complex knots are expected to occupy a larger portion 


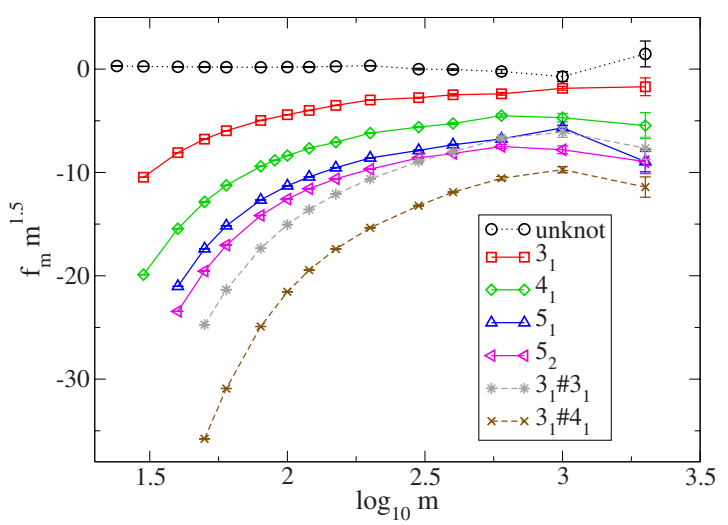

FIG. 5. (Color online) Plot of $f_{m} m^{1+\Delta}$ vs $\log _{10} m$, with $\Delta=1 / 2$. The data tend to a constant for large $m$, which, as discussed in the text, is consistent with a correction-to-scaling exponent of $\Delta \approx 0.5$.

of the polymer. In Table III, we list our estimates of $\bar{A}$ and $\bar{B}$, obtained by means of linear fits to data in the form $f_{m} m^{1.5}$ vs $m^{-0.5}$. The values of $\bar{B}$ are almost two orders of magnitude larger than those of $\bar{A}$, explaining the fitted (effective) leading exponent $\Delta \approx 1$.

\section{KNOTS COMPETITION}

In this section, we discuss entropic competition between knotted polymers in the context of ELPs. The idea of entropic competition between polymers with various constraints was introduced in Ref. [25]. as a direct way to estimate polymer entropic exponents from canonical simulations. This idea is sketched in Fig. 6 and can be implemented in various ways. One can consider, for instance, a polymer loop divided in two sides by a wall [Fig. 6(a)]; the two sides exchange monomers via sufficiently small holes such that the knots cannot pass through. The exchange can also occur through a fictitious "wormhole" [25], as shown in Fig. 6(b). The polymers at the two sides of the wall or those exchanging monomers through the wormhole do not interact with each other. When exchanging monomers the length of each loop fluctuates, while the total length is fixed to a constant $L$. The method [25] is based on the analysis of the equilibrium distribution of lengths of the two sides. For ordinary polymers one expects that the length $l$ of one polymer ring is distributed according to

TABLE III. Fits of $\bar{A}$ and $\bar{B}$, assuming $\Delta=1 / 2$ in Eq. (22).

\begin{tabular}{ccc}
\hline \hline Knot type & $\bar{A}$ & $\bar{B}$ \\
\hline $3_{1}$ & -0.78 & -33 \\
$4_{1}$ & -1.9 & -63 \\
$5_{1}$ & -3.5 & -73 \\
$5_{2}$ & -4.1 & -80 \\
$3_{1} \# 3_{1}$ & -1.4 & -130 \\
$3_{1} \# 4_{1}$ & -3.3 & -173 \\
\hline \hline
\end{tabular}
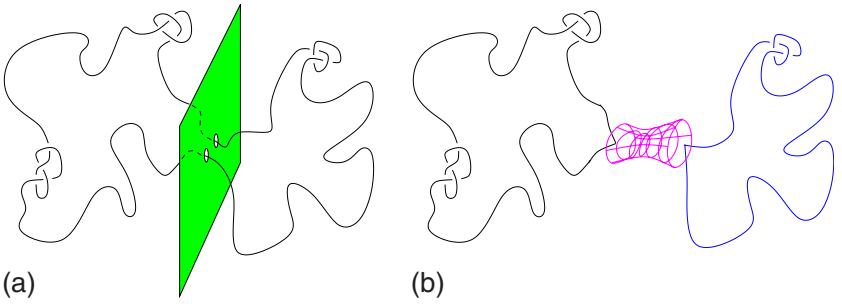

(b)

FIG. 6. (Color online) (a) Sketch of an entropic competition: a portion of the ring polymer, with two knots, is constrained to stay on the left half-space (holes are small enough to forbid more than one monomer at a time to pass), the remaining part has one knot and is on the other side of the wall. The total length $N$ of the chain is constant but the lengths $m$ and $N-m$ of the two subchains can fluctuate. (b) The virtual version (without the wall) of the same competition: the two polymers swim in separate dilute solutions and are coupled via a "wormhole" trough which they can exchange a monomer (hence not a knot) at a time.

$$
p(l) \sim Z_{l}^{(1)} Z_{L-l}^{(2)} \sim \mu^{L} l^{\theta_{1}}(L-l)^{\theta_{2}},
$$

where the two $Z$ 's are the loop partition functions given in Eq. (1). The main point is that the dependence on $\mu$ in Eq. (23) is irrelevant as $L$ is fixed, whereas from the analysis of the shape of the probability $p(l)$ as a function of $l$ it is possible to fit the values of the entropic exponents $\theta_{1}$ and $\theta_{2}$ of the two loops [25].

\section{A. Entropic competition without a wall}

We first consider the case depicted in Fig. 6(b), and we discuss a few representative examples. If the two loops both have negative entropic exponents $\left(\theta_{1}, \theta_{2}<0\right)$, then one expects a $p(l)$ as depicted in Fig. 7(a) (thick line and shaded area), whereas the case $\theta_{1}, \theta_{2}>0$ is depicted in Fig. 7(b) [same notation; in these figures, for convenience we show the distribution $p(l / L)$, which is just a rescaling of $p(l)]$. The thin lines in Figs. 7(a) and 7(b) show sketches of finite- $L$

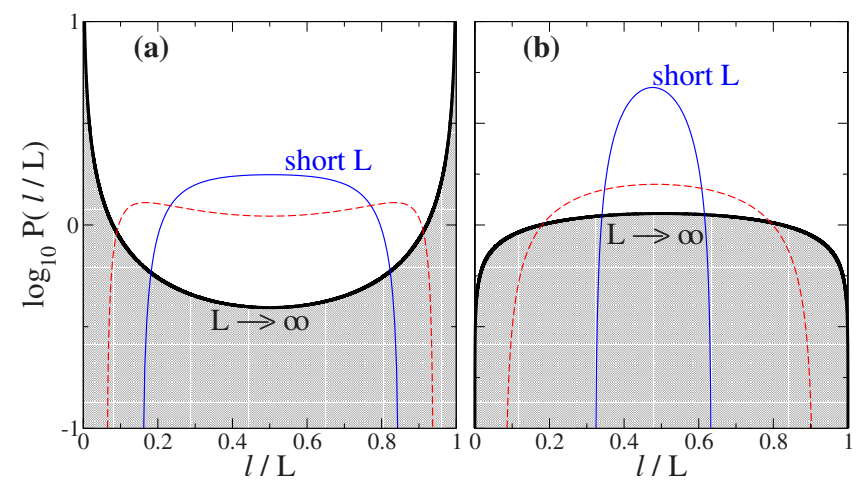

FIG. 7. (Color online) Examples of probability distributions of loop lengths for two polymer loops exchanging monomers as in Fig. 6(b), in the case of negative (a) and positive (b) entropic exponent $\theta$ for both loops. In (a) the competition is between two $3_{1}$ knots, the thick line (boundary of the shaded area) is the distribution for the limit of long $L$ while the other ones are for two short $L$ 's. In (b) the competition is between a $3_{1} \# 3_{1}$ knot and a $3_{1} \# 4_{1}$ knot, with the same notation. 
distributions of $p(l)$ for increasing $L$ : particularly interesting is the scenario depicted in Fig. 7(a), which shows a drastic change of the shape of the distribution from a finite $L$ to the limit $L \rightarrow \infty$. We will discuss here how some of these features can be understood from the analysis of the stored length densities $\rho_{m}^{(1)}$ and $\rho_{m}^{(2)}$ of the competing loops.

Let us consider $p(m)$, the probability of finding $m$ monomers in one of the two entropically competing ELP loops. This quantity scales as

$$
p(m) \sim \widetilde{Z}_{m}^{(1)} \widetilde{Z}_{N-m}^{(2)},
$$

where the two $\tilde{Z}_{s}$ are the partition functions of the two competing ELPs at fixed monomer numbers $m$ and with fluctuating lengths. To find the most probable value of the monomer number $m^{*}$ observed in the entropic competition setup, we maximize the entropy

$$
S_{m}=k_{B} \log \widetilde{Z}_{m}^{(1)}+k_{B} \log \widetilde{Z}_{N-m}^{(2)}
$$

( $k_{B}$ is the Boltzmann constant). The partition functions of ELPs in Eq. (3) are expressed as a sum over all lengths 0 $\leq m \leq l$. The sum is however dominated by a characteristic value of $l^{*}(m)$ obtained from the condition

$$
\left.\frac{\partial \widetilde{Z}_{m, l}}{\partial l}\right|_{l=l^{*}(m)}=0
$$

where we defined

$$
\widetilde{Z}_{m, l} \equiv\left(\begin{array}{c}
m \\
l
\end{array}\right) u^{l} Z_{l}
$$

Now assuming that $\widetilde{Z}_{m}$ is dominated by a single value of $l^{*}(m)$ we can compute the total derivative in $m$ of $\log \widetilde{Z}_{m}$ as

$$
\begin{aligned}
\frac{d \log \tilde{Z}_{m}}{d m} & \approx \frac{d \log \tilde{Z}_{m, l^{*}(m)}}{d m}=\frac{\partial \log \tilde{Z}_{m, l^{*}(m)}}{\partial m} \\
& =\frac{d}{d m} \log \frac{m !}{\left[m-l^{*}(m)\right] !}=-\log \rho_{m} .
\end{aligned}
$$

In this derivation we used Eq. (26), so in the total derivative with respect to $m$ we can ignore the $m$-dependence coming from $l^{*}(m)$. Combining Eqs. (25) and (28) we find that the extremum of the entropy $S_{m}$ of the competing rings is given by the value of $m^{*}$ for which

$$
\rho_{m^{*}}^{(1)}=\rho_{N-m^{*}}^{(2)}
$$

To find $m^{*}$ one can plot $\rho_{m}^{(1)}$ and $\rho_{N-m}^{(2)}$ vs $m$ and $N-m$ in the same graph: each intersection point between the two curves is an extremum of $S_{m}$. To decide whether this is a local maximum or minimum one analyzes the second derivative

$$
\left.\frac{d^{2} S_{m}}{d m^{2}}\right|_{m^{*}} \simeq-\frac{1}{\rho_{m^{*}}^{(1)}}\left[\frac{d \rho_{m}^{(1)}}{d m}-\frac{d \rho_{N-m}^{(2)}}{d(N-m)}\right]_{m^{*}} .
$$

In Fig. 8, we show some plots of the stored-length densities for the two competing loops containing knots. The two loops have a total number of monomers equal to $N=2000$ and the data are those shown in Fig. 4, but now plotted as

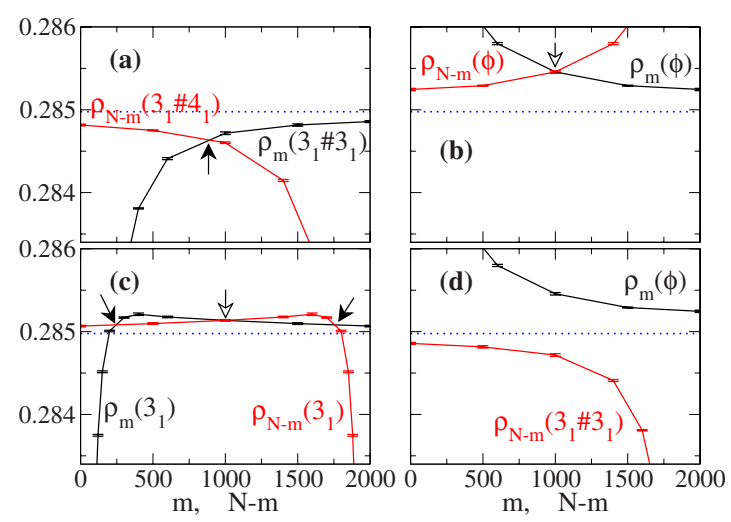

FIG. 8. (Color online) Plot of stored-length densities vs $m$ and $N-m$ in the entropic competition setup, for four different knotted chains: (a) $3_{1} \# 3_{1}$ vs $3_{1} \# 4_{1}$, (b) unknot vs unknot, (c) $3_{1}$ vs $3_{1}$, and (d) unknot vs $3_{1} \# 3_{1}$. The intersection points of the densities are highlighted by arrows and correspond to local maxima (filled arrows) or minima (empty arrows) of the total entropy of the two competing loops. Horizontal dotted lines indicate $\rho_{\infty}$.

function of $m$ and $N-m$. As seen in the previous section, the stored length density can be nonmonotonic in $m$ for some knotted configurations, which can produce various scenarios where up to three intersection points are possible.

Figure 8(a) shows the example of two competing double knots. In this case there is a single intersection point and the analysis of the first derivatives of $\rho$ shows that this point is a local maximum for the entropy [Eq. (30)]. The probability distribution of monomers (or lengths in the canonical setup) will have a single maximum at some intermediate $m^{*}$, as shown in the example of Fig. 7(b). In the case of two unknotted loops [Fig. 8(b)] the intersection point $m^{*}$ is a minimum for the entropy, hence the probability distribution for $m$ will be maximal at the edges and minimal at $m^{*}$, as for the thick line in Fig. 7(a). The most interesting case is that of competition between loops with nonmonotonic $\rho$ 's. This case is illustrated in Fig. 8(c). The three intersection points are a central local minimum of the entropy enclosed by two local maxima. The probability distribution of lengths is like that depicted as a dashed line in Fig. 7(a). It is easy to see that if the total number of monomers decreases (this corresponds to shift one of the two $\rho$ 's along the horizontal axis) there will be only one intersection point. This generates a probability distribution with a single maximum for $m^{*}$ [thin dense line in Fig. 7(a)]. Interestingly, the length distributions obtained from Monte Carlo simulations [25] of competing off-lattice flexible rings with simple knots give, for sizes up to 200 monomers, concave distributions, contrary to the expectations of negative $\theta$ 's from the conjecture of Ref. [13], which would instead correspond to a convex (i.e., with a minimum in the middle) shape. The nonmonotonicity in $m$ of the stored-length density $\rho_{m}$ explains this drastic change in behavior in finite-size data.

To complete the discussion, we consider next an example where no intersection point is present [Fig. 8(d)]. In this case one has to resort to the full form of $S_{m}$ : from the scaling of partition functions of SAPs, the probability of a state with $m$ monomers on the side with no knots is expected to scale as 
$m^{-3 \nu} \sim m^{-1.76}$ with a cutoff at $m \lesssim N$. It implies that the average length of the unknotted subchain $\langle m\rangle_{N} \sim N^{\alpha} \sim N^{0.24}$ is weakly scaling with $N$, and at least in this case the competition is clearly in favor of the side with knots. This reminds us that the full statistics given by $S_{m}$ would often be necessary to compute average quantities, and that the maxima are only indicative elements. Nevertheless, we have seen that the density of stored length is a useful quantity for understanding the basic properties of the entropy of competing knotted chains. In particular, knowing it and its short- $N$ features helps to interpret the numerical results and to distinguish preasymptotic scalings from asymptotic ones.

\section{B. Entropic competition with a wall}

Let us finally go back and reconsider briefly the entropic competition of knots divided by a wall, as in Fig. 6(a). The main difference is that the basic exponent of the unknotted chain should be $\theta_{s}=-d \nu+\sigma_{2}^{\prime}$. The additional index $\sigma_{2}^{\prime}$ is connected to the constraint of having a monomer of a loop confined close to a hard surface. The formula is an application of Duplantier's general theory of polymer networks $[43,44]$. We use this theory also to extract $\sigma_{2}^{\prime}$ from the data in Ref. [45], obtaining $\sigma_{2}^{\prime} \approx-0.95$. This means that $\theta_{s} \approx-2.71$. Again the full zoology of possible competitions could be simply discussed by repeating the above reasoning, once data of $\rho_{m}$ for ELPs close to a wall are generated. We reserve this investigation for a future work. Let us just note that the condition $\theta_{s}+\pi_{k}>0$, associated with a single maximum of the entropy $S_{m}$ at $1 \ll m^{*} \ll N$, is now met for a minimal number of prime knots $\pi_{k}=3$ per loop, i.e., one more than we needed in the case without the wall. Thus, the wall separating the chains has somewhat the effect of repelling entropically also the knots.

\section{CONCLUSIONS}

In this paper, we studied the scaling properties of a class of polymers, which we have referred to as ELPs. These polymers can accumulate stored length along their backbone, by lifting the self-avoidance condition for neighboring monomers of monomers. The length $l$ of their backbone fluctuates, whereas the total number of monomers $m$ is fixed. Differently from true grand canonical polymers, however, the backbone length is bounded to $l \leq m$, and fluctuates around $\langle l\rangle$ with fluctuations $\sim \sqrt{m}$.

ELPs were used in the past to study the dynamics of polymer melts [22] and pore translocation dynamics [21], but their equilibrium behavior has received little attention. In this work, we used ELPs to investigate entropic exponents of knotted polymer rings. The calculation of polymer entropic exponents from classical Monte Carlo simulations of canonical self-avoiding rings is rather cumbersome: one has either to employ complex grand canonical sampling, or to resort to the so-called atmospheres method [19], or entropic competition methods [25]. ELPs are well suited to this type of problem. First, the underlying Monte Carlo dynamics can be based solely on local moves (thus conserving the knot topology) and the possibility of accumulating length along the backbone facilitates the sampling of different configurations compared to canonical self-avoiding rings. The ELP explores new configurations through sliding moves along the backbone. Second, we have shown that there is a natural variable associated to ELPs which is the stored length density $\rho_{m}$ [see Eq. (2)], which measures the average fraction of monomers accumulated on the backbone. We have derived an expansion for $\rho_{m}$ in the limit $m \rightarrow \infty$, where $\rho_{m}$ converges to a value that depends on the connectivity constant of the ordinary lattice polymers. The next leading behavior is of the order $\theta / \mathrm{m}$, with $\theta$ the entropic exponent of the polymers. This allows one to estimate entropic exponents from the scaling analysis of $\rho_{m}$. As examples of application of this, we estimated entropic exponents of swollen polymers in $d=2$ and $d=3$, and of polymers with various types of knots. Comparing the results with conjectured values of these exponents, we find a clear agreement at least for the simplest knot studied. For more complex knots the agreement is only marginal, due to finite-size effects quickly increasing with the knot complexity.

One of the advantages of the stored length analysis is that correction-to-scaling effects are directly visible in $\rho_{m} \mathrm{vs} 1 / \mathrm{m}$ plots as they appear as deviations from a linear scaling behavior. Our analysis showed that finite-size effects become stronger with the knot complexity and with the number of knots. Similar result have been observed by Janse van Rensburg and Rechnitzer [19]. These authors estimated the connectivity constant and entropic exponent of lattice polymers via the atmospheres method [18], where, roughly speaking, atmospheres are the loci where the polymer can be expanded and contracted. Interestingly, there is a similarity between the scaling of the average atmospheres and that of the storedlength density of ELPs discussed in this paper.

With simulations of ELPs we have shown how important are corrections to scaling in the statistics of knotted polymers: their equilibrium properties in entropic competition can be understood from coexistence diagrams of stored lengths of ELPs. The nonmonotonicity of the stored length density as function of $1 / L$ explains some features of the competing rings observed in canonical Monte Carlo simulations [25] which were poorly understood before.

Summarizing, the elastic lattice polymer is a simple model sharing critical exponents with the self-avoiding walk, but it has an additional "elastic" degree of freedom in its fluctuating length, which offers numerical advantages and additional theoretical tools to derive critical exponents of polymers. Thus, the ELP is a valid alternative to classical lattice models for studies in polymer physics.

\section{ACKNOWLEDGMENTS}

G.B. and M.B. acknowledge hospitality from the Institute of Theoretical Physics at the K. U. Leuven and financial support from the Research Foundation-Flanders (FWO) Grant No. G.0311.08. M.B. acknowledges financial support from the University of Padua (Progetto di Ateneo $n$. CPDA083702). 
[1] A. Pelissetto and E. Vicari, Phys. Rep. 368, 549 (2002).

[2] P.-G. de Gennes, Scaling Concepts in Polymer Physics (Cornell University Press, Ithaca, USA, 1979).

[3] J. des Cloizeaux and G. Jannink, Polymers in solution: Their modeling and structure (Clarendon Press, 1990).

[4] C. Vanderzande, Lattice Models of Polymers (Cambridge University Press, Cambridge, England, 1998).

[5] N. Clisby, Phys. Rev. Lett. 104, 055702 (2010).

[6] B. Berg and D. Foerster, Phys. Lett. B 106, 323 (1981) ; C. Aragão de Carvalho, S. Caracciolo, and J. Fröhlich, Nucl. Phys. B 215, 209 (1983).

[7] B. Nienhuis, Phys. Rev. Lett. 49, 1062 (1982).

[8] F. Seno and A. L. Stella, J. Phys. (France) 49, 739 (1988).

[9] T. Ishinabe, Phys. Rev. B 39, 9486 (1989).

[10] N. Madras and A. D. Sokal, J. Stat. Phys. 50, 109 (1988).

[11] R. Brak, A. L. Owczarek, and T. Prellberg, J. Phys. A 26, 4565 (1993).

[12] P. Grassberger and R. Hegger, J. Phys. (France) 5, 597 (1995).

[13] E. Orlandini et al., J. Phys. A 29, L299 (1996).

[14] E. Orlandini, M. C. Tesi, E. J. Janse van Rensburg, and S. G. Whittington, J. Phys. A 31, 5953 (1998).

[15] S. Caracciolo, M. S. Causo, and A. Pelissetto, Phys. Rev. E 57, R1215 (1998).

[16] I. Jensen and A. J. Guttmann, J. Phys. A 32, 4867 (1999).

[17] I. Jensen, J. Phys. A 37, 5503 (2004).

[18] A. Rechnitzer and E. J. Janse van Rensburg, J. Phys. A 35, L605 (2002).

[19] E. J. Janse van Rensburg and A. Rechnitzer, J. Phys. A: Math. Theor. 41, 105002 (2008).

[20] N. Clisby, R. Liang, and G. Slade, J. Phys. A: Math. Theor. 40, 10973 (2007).

[21] J. K. Wolterink, G. T. Barkema, and D. Panja, Phys. Rev. Lett. 96, 208301 (2006).

[22] A. van Heukelum and G. T. Barkema, J. Chem. Phys. 119, 8197 (2003).

[23] M. Baiesi, E. Orlandini, and A. L. Stella, Phys. Rev. Lett. 87, 070602 (2001).

[24] H.-P. Hsu, W. Nadler, and P. Grassberger, Macromolecules 37, 4658 (2004).

[25] R. Zandi, Y. Kantor, and M. Kardar, ARI Bull. Instanbul Tech. Univ. 53, 6 (2003).

[26] E. J. Janse van Rensburg and S. G. Whittington, J. Phys. A 23,
3573 (1990).

[27] E. J. Janse van Rensburg, E. Orlandini, D. W. Sumners, M. C. Tesi, and S. G. Whittington, J. Knot Theory Ramif. 6, 31 (1997)

[28] T. Deguchi and K. Tsurusaki, Phys. Rev. E 55, 6245 (1997).

[29] H. Matsuda, A. Yao, H. Tsukahara, T. Deguchi, K. Furuta, and T. Inami, Phys. Rev. E 68, 011102 (2003).

[30] R. Metzler, A. Hanke, P. G. Dommersnes, Y. Kantor, and M. Kardar, Phys. Rev. Lett. 88, 188101 (2002).

[31] B. Marcone, E. Orlandini, A. L. Stella, and F. Zonta, J. Phys. A: Math. Theor. 38, L15 (2005).

[32] E. Orlandini and S. G. Whittington, Rev. Mod. Phys. 79, 611 (2007).

[33] M. Baiesi, E. Orlandini, and A. L. Stella, Phys. Rev. Lett. 99, 058301 (2007).

[34] M. Baiesi, E. Orlandini, and S. G. Whittington, J. Chem. Phys. 131, 154902 (2009).

[35] M. Baiesi, E. Orlandini, and A. L. Stella, e-print arXiv:1003.5134, J. Stat. Mech.: Theory Exp. (to be published).

[36] A. Stasiak, V. Katritch, J. Bednar, D. Michoud, and J. Dubochet, Nature (London) 384, 122 (1996).

[37] V. V. Rybenkov, N. R. Cozzarelli, and A. V. Vologodskii, Proc. Natl. Acad. Sci. U.S.A. 90, 5307 (1993).

[38] J. Arsuaga, M. Vazquez, P. McGuirk, S. Trigueros, D. Sumners, and J. Roca, Proc. Natl. Acad. Sci. U.S.A. 102, 9165 (2005).

[39] W. R. Taylor, Nature (London) 406, 916 (2000).

[40] R. C. Lua and A. Y. Grosberg, PLoS Comput. Biol. 2, 0350 (2006)

[41] Y. Burnier, J. Dorier, and A. Stasiak, Nucleic Acids Res. 36, 4956 (2008).

[42] M. Henkel and G. Schutz, J. Phys. A 21, 2617 (1988).

[43] B. Duplantier, J. Stat. Phys. 54, 581 (1989).

[44] L. Schäfer, C. von Ferber, U. Lehr, and B. Duplantier, Nucl. Phys. B 374, 473 (1992).

[45] D. S. Gaunt and S. A. Colby, J. Stat. Phys. 58, 539 (1990).

[46] M. Baiesi and E. Orlandini (unpublished).

[47] In principle we should consider the connectivity constant $\mu_{0}$ of the ensemble of configurations with a given knot, which is slightly smaller than $\mu$. However, their estimated difference [46] is smaller than the numerical error for $\mu$. 